\title{
PERFORMANCE ANALYSIS OF IMAGE FILTERING ALGORITHMS FOR MRI IMAGES
}

\author{
Sivasundari .S, R. Siva Kumar ${ }^{2}$, M.Karnan ${ }^{3}$ \\ ${ }^{\text {I}}$ P.G scholar, Department of Computer and Communication, Tamilnadu College of Engineering, Coimbatore, \\ TamilNadu, India \\ ${ }^{2}$ Professor, Department of Information Technology, Tamilnadu College of Engineering, Coimbatore, TamilNadu, India \\ ${ }^{3}$ Professor \& Head, Department of Computer Science and Engineering, Tamilnadu College of Engineering, \\ Coimbatore, TamilNadu, India
}

\begin{abstract}
In Image Processing, the digital images are much sensitive to noise which results due to the Image Acquisition errors and transmission errors.MRI images captured usually are prone to speckle noise, Gaussian noise, salt and pepper noise etc., Image filtering algorithms are applied over the noisy images to remove the noise and preserve the image details. In this work three different filtering algorithms such as Median filter $(M F)$, Weiner filter $(W F)$ and Center Weighted Median filter $(C W M)$ are used to remove the noise present in the MRI images. The performance of these filters are compared using the statistical parameters such as Mean Square Error(MSE) and Peak Signal to Noise Ratio(PSNR).The study shows that the Weiner filters reconstructs a high quality image than Median and Center weighted Median filter.
\end{abstract}

Keywords: Median filter (MF), Weiner filter (WF), Center Weighted Median filter (CWM), Mean Square Error (MSE) and Peak Signal to Noise Ratio (PSNR).

\section{INTRODUCTION}

In image processing, it is important to obtain quality image for facilitating the image classification accuracy. As the poor image quality is an obstacle for efficient image processing. There is a need for the noise removal in medical images. In this work MRI images are used for the experiment.

Magnetic Resonance Imaging are now becoming the most indispensible medical imaging tool used for analysis of different parts of the human brain.MRI produces the detailed information of the internal parts of the brain which can be used for the radiologists to detect the presence of tumors in the patient's brain. The scan does not penetrate the adjacent healthy cells and thus preserves to be safest diagnostic tool. However one of the primary problems with MRI images are the presence of added unwanted noises like speckle noise, Gaussian noise,salt,Rician noise ,salt and pepper noise etc.,

These noise have much influence on the quality of the image which in turn degrades the performances of Feature Extraction, Reduction and Classification of the processed images. The noise have to be removed before these processing stages. Many image filtering algorithms are available in the literature.

This paper evaluates the three filtering algorithms for different noise types. Simulation is done with the MATLAB R2010a.The organization of this paper is as follows. Section 2 is described with image filtering methods. Section 3 discuss about the Experiment results and evaluation and finally Section 4 gives conclusion.

\section{FILTERING METHODS}

Noise is the unwanted variations or fluctuations of the image capture. These variations are caused due to errors in the sensors or data transmission which corrupts the image details either by causing brightness or frequency changes. Speckle noise are caused due to transmission errors. Rician noise are caused due to the Gaussian noise in the original frequency domain measurements [1]. The amplifier produces a standard model of noise which is additive, Gaussian. These noise are independent at each pixel and its intensity. Amplifier noise is a cause of the "read noise" of an image sensor that is, having constant noise level in dark areas of the image [2]. A salt-andpepper noise will have dark pixels and bright pixels alternate bright and dark regions [2].Speckle noise is a granular noise degrades the quality due to transmission errors [3].

Conventionally used simplest methods of linear filtering algorithms include mean filters. The filtering is performed by using a mask over every pixel component in the image. Then by averaging the pixels which falls under the mask results in a single pixel to be studied. This technique also called as averaging filter. The main drawback of this filter is that it is poor in edge preserving.

\subsection{Median Filter}

The Median filter is advantageous over mean filter and its a non-linear filtering technique, helps removing noise. Convolution methods like smoothing filters reduces noise but blurs the edge features. In order to preserve the edge features and [4] sharpness of the image we go for Median Filters. It 
does excellent job in rejecting impulse noise, in which some individual pixels have extreme values. In Median filtering, for each pixel, the pixel values in the neighborhood window are organized according to the intensity and the middle value(Median) becomes the new value for the central pixel.i.e., each pixel is set to an 'average' of the pixel values in the neighborhood pixels rather than the mean. This method can be repeated if necessary since it causes only less blur of edges. Median filter is less sensitive than the mean filter to extreme values .The median filter (M) can be implemented by the formula:

$$
\mathrm{M}\left(\mathrm{P}_{l} \ldots \mathrm{P}_{N}\right)=\operatorname{Median}\left(\left\|\mathrm{P}_{l}\right\|^{2} \ldots \ldots .\left\|\mathrm{P}_{N}\right\|^{2)}-\right.
$$

This is an optimal filter much suitable for de-noising images of various noise types[5].Causes no changes in the shift boundaries and no reduction in contrast Causes no changes in the shift boundaries and no reduction in contrast and proved to be more robust in the presence of noise.

\subsection{Weiner Filter}

Weiner filtering carry out an optimal tradeoff between inverse filtering and noise smoothing. It removes additive noise and deblurring concurrently [6]. This proves to be optimal in reducing the overall Mean Square Error (MSE).The operation involves two parts. One is inverse filtering and the other part is noise smoothing. Wiener filters belong to a kind of optimum linear filters with the noisy data as input which involves the calculation of difference between the desired output sequences from the actual output.

Main purpose of this statistical approach is to diminish the noise present in a signal by comparing it with an estimation of the desired noiseless signal. Wiener filters are characterized by an assumption that signal and (additive) noise are stationary linear random processes and their spectral characteristics are calculated. The Performance can be measured using Minimum Mean-Square Error.

The Wiener filter is implemented as:

$$
W(m, n)=\frac{H^{*}(m, n) P_{S}(m, n)}{|H(m, n)|^{2} P_{S}(m, n)+P_{n}(m, n)}
$$

Dividing through by $P_{s}$ :

$$
W(m, n)=\frac{H^{*}(m, n)}{|H(m, n)|^{2}+\frac{P_{n}(m, n)}{P_{S}(m, n)}}
$$

Where

$\mathrm{H}(\mathrm{m}, \mathrm{n})=$ Degradation function ,

$\mathrm{H}^{*}(\mathrm{~m}, \mathrm{n})=$ Complex conjugate of degradation function,

$\mathrm{P}_{\mathrm{n}}(\mathrm{m}, \mathrm{n})=$ Power Spectral Density of Noise ,

$\mathrm{P}_{\mathrm{s}}(\mathrm{m}, \mathrm{n})=$ Power Spectral Density of un-degraded image.

The term $\mathrm{P}_{\mathrm{n}} / \mathrm{P}_{\mathrm{s}}$ can be interpreted as the reciprocal of the signal-to-noise ratio [5].The statistical parameters Mean Square Error (MSE) and Peak Signal-to-Noise ratio (PSNR) are used to evaluate the enhancement performance.

\subsection{Center Weighted Median Filter (CWM)}

The CWM filter is an extension of Weighed median filter. Median filters can remove the noise by preserving the edges and sharpness which is suitable to reduce salt and pepper noise. In Weighted median filter, the weights of the filter are assigned based on intensity value of pixels in the MRI image. The weights used here are $0,0.1,0.2$ and 0.3.Initially the weight of the pixel is set to 0 ,if the pixel intensity is 0 .The weight assigned is 0.1 for the pixel intensity range between 1 100 and 0.2 for pixel intensity range 101-200 else 0.3 . The weights set are multiplied with pixel intensity and Median Filter is applied. This process is called Weighted Median Filter.

If the center value of each window is set more weight, then its Center Weighted Median filters (CWM). The CWM filters are represented by two important parameters such as window size and center weight which influence the quality of filter. The center value is assigned large weight i.e. $\mathrm{W}(0,0)=2 \mathrm{~N}+1$, where $\mathrm{N}$ is a non-negative integer, if $\mathrm{N}>=0$ then $\mathrm{W}(\mathrm{i}, \mathrm{j})=1, \mathrm{i}$ and $\mathrm{j}$ not equal to 0 .

The performances of CWM are better analyzed by authors $[7,8]$.The value of weight must chosen carefully as it drives the performance of the filter. The image characteristics and type of noise should be considered while selecting the weight. The larger weight provides better result in preserving image and edge details but worse in noise removal [9].

\section{EXPERIEMENTS AND RESULTS}

These filtering algorithms has been tested with various types of noisy images implemented by using MATLAB 7.1.0.. The experimental results are tested in Intel Pentium IV $2.4 \mathrm{GHz}$ processor with $256 \mathrm{MB}$ RAM. Performance of the Median filter, Center Weighted Median filter, and Weiner filters are analyzed and evaluated.

Table -1: shows the performance of listed filters

\begin{tabular}{|l|l|l|l|}
\hline S.no & Filters & MSE & PSNR \\
\hline 1 & $\begin{array}{l}\text { Median } \\
\text { Filter }\end{array}$ & 148949 & $\mathbf{7 . 1 9 9 1}$ \\
\hline 2 & $\begin{array}{l}\text { CWM } \\
\text { Filter }\end{array}$ & $2.7819 \mathrm{e}+006$ & 16.312 \\
\hline 3 & $\begin{array}{l}\text { Weiner } \\
\text { Filter }\end{array}$ & $8.3636 \mathrm{e}+003$ & 17.813 \\
\hline
\end{tabular}



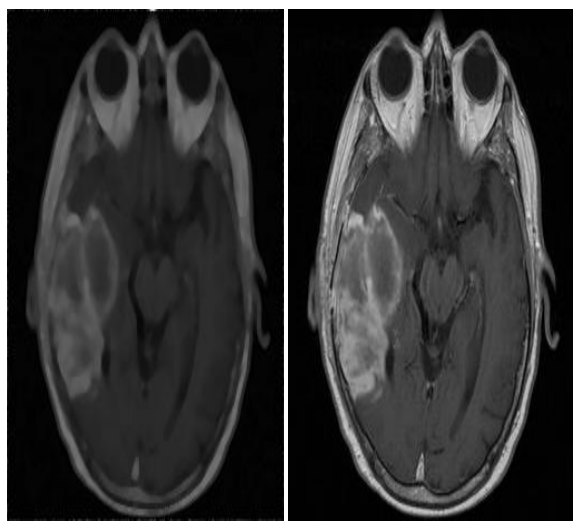

Fig -1 Input Image

Fig -2 CWM Filtered
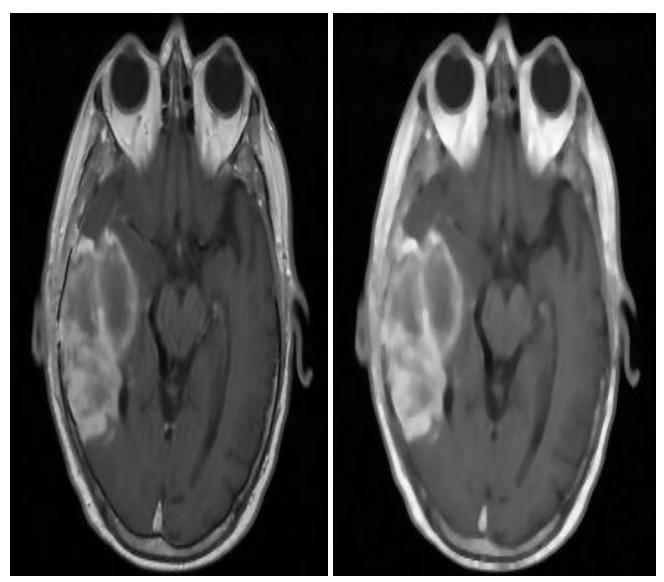

Fig -3 Median Filtered Fig -4 Weiner Filtered

[4]. Bhausaheb Shinde et al,2012," Study of Noise Detection and Noise Removal Techniques in Medical Images", I.J. Image, Graphics and Signal Processing, 2, 51-60.

[5]. Dr.G.Padmavathi et al ,2009," Performance analysis of Non Linear Filtering Algorithms for underwater images", (IJCSIS) International Journal of Computer Science and Information Security, Vol.6, No. 2,

[6]. N.Rajalakshmi and V.Lakshmi Prabha,2009," Automated Classification of brain MRI using color converted k-means clustering segmentation and application of different kernel functions with multi-class SVM", 1st Annual International Interdisciplinary Conference, AIIC 2013, 24-26.

[7]. E. Ben George et al, 2012," MRI Brain Image Enhancement Using Filtering Techniques", International Journal of Computer Science \& Engineering Technology (IJCSET), ISSN: 2229-3345 Vol. 3 No. 9

[8]. Tong Sun, 1995,"Analysis of two dimensional center weighted median filters", Multidimensional systems and signal processing, 6,159-172.

[9]. J.M. Waghmare, 2013,"Removal of Noises In Medical Images By Improved Median Filter", The International Journal Of Engineering And Science (IJES), ISSN(p): 2319 1805.

[10]. Bhausaheb Shinde et al, 2012," Apply different techniques to remove the speckle noise using medical images", International Journal of Engineering Research and Applications (IJERA) ISSN: 2248-9622.

\section{CONCLUSIONS}

In this paper image filtering algorithms are implemented on MRI images to remove the different types of noise that are either present in the image during acquisition or injected in to the image during transmission. MRI images when captured usually have Gaussian noise and speckle noise. In this work, three different image filtering algorithms are compared for the noisy images. The performances of the filters are measured using the Peak Signal to Noise Ratio (PSNR) and Mean Square Error (MSE). The Weiner filter gives desirable results with large PSNR value ensuring high image enhancement.

\section{REFERENCES}

[1]. M. N. Nobi and M. A. Yousuf, 2011," A New Method to Remove Noise in Magnetic Resonance and Ultrasound Images" Journal of Scientific Research,, J. Sci. Res. 3 (1), 81-89.

[2]. Shi Zhong, 2000,"Image Denoising using Wavelet Thresholding and Model Selection", Image Processing, International Conference on, Volume: 3, 10-13 Sept. 2000 Pages: 262.

[3]. Suresh Kumar et al, 2010,'Performance Comparison of Median and Wiener Filter in Image De-noising", International Journal of Computer Applications (0975 8887) Volume 12- No.4 